\title{
ANÁLISIS DE LA GESTIÓN DE LA POLÍTICA EDUCATIVA DEL MINISTERIO DE EDUCACIÓN DE CHILE DESDE LA PERCEPCIÓN DE ESTUDIANTES, DOCENTES Y DIRECTIVOS
}

\author{
Katherine López Arias \\ Universidad de Aconcagua (Chile)
}

\begin{abstract}
RESUMEN: El presente estudio busca conocer las percepciones de los agentes integrantes acerca de las políticas de mejoramiento de la calidad de la gestión escolar de un establecimiento educacional de la Comuna de Punta Arenas. En este contexto, se aborda principalmente las políticas educativas y el concepto de calidad en Educación. Se trata de una investigación, con un diseño no experimental de carácter descriptivo, abordada desde la complementariedad paradigmática, y que busca analizar la gestión escolar en el marco de la aplicación de las políticas educativas emanadas del Ministerio de Educación de Chile (MINEDUC). Para el enfoque cuantitativo se emplea un cuestionario de estudiantes y otro de docentes, mientras que la parte cualitativa se realiza mediante una entrevista a los agentes integrantes (estudiantes, docentes, directivos y apoderados). A partir de los resultados se concluye que, efectivamente, las políticas ministeriales son parte de la definición de políticas del establecimiento establecidas en el Plan de Desarrollo Institucional (PDI). Sin embargo, en la práctica, se evidencia falta de participación de todos los actores de la comunidad educativa, ya que el Plan Estratégico Institucional no da cuenta de los intereses de la comunidad.
\end{abstract}

PALABRAS CLAVE: Gestión de políticas educativas, mejoramiento de la calidad, percepciones.

\section{ANALYSIS OF THE EDUCATIONAL POLICY MANAGEMENT OF THE CHILEAN MINISTRY OF EDUCATION BASED ON TEACHER, STUDENT AND LEADER PERCEPTION SURVEYS}

\footnotetext{
ABSTRACT: This research seeks to know the perceptions of integrating agents about the school management quality policies applied at a High School in the city of Punta Arenas, Magellan and Antarctic Region. Thus, the Chilean educational policies and the concept of quality are dealt with. It is non-experimental, quantitative-qualitative descriptive study, approached from
} 
a mixed paradigm that analyzes this high school's management regarding the educational policies set forth by the Chilean Ministry of Education. For the quantitative approach a student and teacher survey is applied, whereas for the qualitative part an interview to the integrating agents (students, teachers, leaders and parents) is carried out. As a result, it is concluded that the ministry's educational policies are integrated in this high school's Institutional Development Plan. However, in practice, not all members of this educational community participate in the decision-making process, as its Strategic Plan does not take the interests of this educational community into account.

KEYWORDS: Educational policy management, quality improvement, perceptions.

Recibido: 28/05/2014

Aceptado: 19/10/2014

\section{INTRODUCCIÓN}

El presente estudio se enmarca dentro de la investigación sobre el ámbito de la gestión escolar. En los últimos años, la investigación en esta área ha aportado nuevos datos que ponen de relieve la importancia de las políticas ministeriales y su implementación, a nivel de los establecimientos educacionales. En este sentido, es importante señalar que las políticas de mejoramiento para lograr una educación de calidad del MINEDUC consisten, específicamente, en la aplicación de programas y proyectos que permitan mejorar la calidad educativa y los resultados del desempeño institucional. Desde el discurso oficial, se plantea la necesidad de abordar la gestión de la institución escolar, de manera integral, pues ésta se traduciría en un elemento básico para generar procesos de mejora continua y así asegurar el mejoramiento de los resultados de aprendizajes de los estudiantes. Sin embargo, desde esta mirada integral, no se ha considerado que cada unidad educativa constituye una realidad distinta, con sus propias visiones, proyectos educativos, problemáticas, y niveles de desarrollo.

De igual manera, aparece como un elemento clave en la problemática del estudio, analizar lo que el discurso oficial señala con respecto a las estrategias de mejoramiento en los ámbitos de gestión educativa, como lo es el Sistema de Aseguramiento de Calidad de la Gestión Escolar (SACGE) y Proyecto Liceos Prioritarios, entre otros, versus lo que realmente acontece en la práctica educativa.

Al respecto, un desarrollo importante en Chile, en materia de gestión educativa, ha sido el desarrollo del Marco de la Buena Dirección (MINEDUC, 2005), documento oficial, que pone el acento en un conjunto de dominios y criterios, incluyendo liderazgo, gestión curricular, gestión de recursos, clima organizacional y convivencia. En este contexto, se concibe al director como pieza clave en el liderazgo del Proyecto Educativo Institucional (PEI), cuyo foco está en mejorar los aprendizajes de los estudiantes y el desempeño institucional en su conjunto.

En consecuencia, y considerando los recursos disponibles para este estudio, se ha seleccionado un liceo, que forma Técnicos de Nivel Medio. Cabe señalar que 
esta unidad educativa depende de la Corporación de Educación, Salud y Atención al Menor de la Comuna de Punta Arenas, que cobija a los establecimientos con mayor vulnerabilidad en la comuna, implementando en ellos los programas de apoyo al mejoramiento institucional del MINEDUC. Es necesario puntualizar que esta unidad educativa atiende a una población escolar mixta, de nivel socioeconómico medio bajo, proveniente de los sectores populares de la ciudad, fundamentalmente de hogares uni-parentales, en los que predominan las madres trabajadoras y/o jefas de hogar. En este contexto, la retención de estudiantes, la calidad educativa y la atención a la diversidad aparecen como una necesidad prioritaria en el establecimiento educacional.

\section{Marco teórico}

En materia de calidad educativa, se tiende a trasladar la promoción de la calidad del mundo empresarial al sistema educativo, lo que obliga a los gobiernos a plantearse la necesidad de mejorar el rendimiento del sistema educativo para así dar respuesta a las demandas socioeducativas (Barrio, 2006). En educación, esta realidad está estrechamente relacionada con la efectividad docente. En este sentido, un estudio relacionado encontró que, en Finlandia, Ontario y Singapur, existen programas institucionalizados para reclutar a los mejores profesores a quienes se les ofrecen programas permanentes de apoyo y desarrollo profesional (Darling-Hammond y Rothman, 2011). Otros recientes estudios sobre los logros académicos de los estudiantes han encontrado que las cualificaciones de los profesores influyen significativamente en el aprendizaje de los estudiantes (Darling-Hammond, 1999).

Con respecto a la experiencia del docente, Darling-Hammond (1999) sostiene que la efectividad docente depende en algún grado de los años de ejercicio de la profesión. También señala que los profesores con menos experiencia son menos efectivos, nivelándose el beneficio de los años de experiencia alrededor de los cinco años. Adicionalmente, señala que los profesores de más edad y que trabajan en establecimientos educacionales, que promueven el perfeccionamiento continuo y el trabajo colaborativo, mejoran su desempeño docente. En este sentido, la colaboración constituye un factor favorecedor de la calidad educativa, como señala Fullan (1993). Por tanto, las instituciones educativas necesitan ofrecer a sus docentes oportunidades para trabajar en forma colaborativa. Esto se vincula estrechamente con el crecimiento y desarrollo personal y profesional que se logran dentro de un contexto de comunidad. La experiencia que se gana en las actividades colaborativas favorece el éxito de las personas. En este sentido, y siguiendo a Morin (1999), se puede asegurar que los profesores efectivos deben ser capaces de crear una verdadera comunidad de aprendizaje al interior de las aulas. Esto está directamente relacionado con el capital decisional, que es cuando los profesores toman decisiones en conjunto con el fin de mejorar la práctica pedagógica.

En términos de calidad educativa, lo anterior significa reclutar profesores altamente comprometidos, bien preparados, dispuestos a perfeccionarse, bien pagados, y vinculados con redes académicas para así maximizar su desarrollo e influenciar positivamente en los aprendizajes de los estudiantes (Fullan y Hargreaves, 2012). De 
esta manera, la calidad y la equidad en las sociedades contemporáneas, constituyen uno de los ejes del debate mundial.

En efecto, en la IX Conferencia Iberoamericana de Educación, celebrada en La Habana, los ministros del área manifestaron claramente que no puede hablarse de un sistema educativo de calidad, si al mismo tiempo no se incluyen programas de equidad con una atención diferenciada, que respondan a la variedad de necesidades que presentan cotidianamente los estudiantes.

Por otra parte, en 1990 la calidad educativa es abordada en la Declaración Mundial sobre Educación para Todos de Jomtien, identificándola como un requisito para alcanzar el objetivo fundamental de la equidad. Una década más tarde, en el Marco de Acción de Dakar (UNESCO, 2000) se declara que el acceso a una educación de calidad es derecho de todos los niños, afirmándose que la calidad está en el centro de la educación, como factor decisivo de la admisión, retención y logro académico.

De acuerdo a la UNESCO (2008), los sistemas educativos pueden fomentar la integración de todos los niños, adolescentes, jóvenes y adultos de todas las edades de diferentes maneras, en particular facilitándoles un acceso equitativo en todas las etapas y cursos de la enseñanza. Al respecto, el modelo de gestión de calidad aplicado a las instituciones educativas, puede concebirse como "un proceso tejido de múltiples dimensiones interrelacionadas entre sí, de forma recursiva" (Morin, 1997, citado en Tobón, Sánchez, Carretero y García, 2004, p. 84), que Ileva implícito la idea de cambio.

Por su parte, el estudio "Un Enfoque Global de la Pobreza y Superación", realizado por la Fundación Nacional (Chile) para la Superación de la Pobreza en 1999, señala que la falta de oportunidades educativas es lo que refuerza el círculo de la pobreza y de la desigualdad. A su vez, los estudios evidencian que los niños y jóvenes de estos hogares pobres, obtienen logros educativos muy inferiores en las pruebas del Sistema de Medición de la Calidad de la Educación (SIMCE) o de la Prueba de Selección Universitaria (PSU), en comparación con estudiantes procedentes de hogares medios.

Sin embargo, como plantea Valdebenito (2011), a través de dichas pruebas sólo se intenta medir la calidad de la educación en términos cuantitativos, es decir, se apunta más bien al producto y no al proceso. En este contexto, la educación en Chile ha avanzado desde una etapa en la que el acceso a las oportunidades educativas y la cobertura de las instituciones era el principal requerimiento, a otra en que la calidad de los aprendizajes y su distribución social son una exigencia prioritaria.

Por ende, es menester destacar que el concepto de educación de calidad, establecido en la Ley General de Educación (LEGE) pone énfasis en el desarrollo integral de los alumnos, y no sólo en el logro de estándares de aprendizaje, entendiendo que el objetivo es brindar una formación que abarque competencias, conocimientos y valores sustentados en la democracia y la solidaridad. Adicionalmente, la distribución de las oportunidades educativas es un factor determinante para las futuras oportunidades laborales, así como para el desarrollo de virtudes cívicas y morales que hacen posible desarrollar una vida social más digna.

A partir de la década de 1990, en el contexto de la reforma educativa se han desarrollado una serie de acciones programáticas, que han tenido como foco el mejoramiento de las condiciones en las cuales se implementa el trabajo escolar en 
los establecimientos con financiamiento público. Así, el Programa de Mejoramiento de la Calidad y Equidad en la Enseñanza Media (MECE-Media), implementado a partir del año 1994, busca mejorar sustantivamente la calidad, equidad y eficiencia de las condiciones, procesos y resultados de la educación media, mejorando las prácticas institucionales para responder mejor al nuevo escenario educativo.

Al respecto, durante el año 1999, el MINEDUC solicitó al Departamento de Ingeniería Industrial de la Universidad de Chile, un estudio de estratificación de los establecimientos de enseñanza media orientado a focalizar acciones programáticas, cuyos resultados, a través del Programa Liceo Para Todos, permitieron focalizar la política educativa en 350 liceos que presentaban los resultados educativos más bajos y atendían a la población más vulnerable. El diseño de este programa, que tuvo como objetivo disminuir la deserción escolar y mejorar la calidad de los aprendizajes en los liceos focalizados, definió una propuesta para los liceos que requerían de estrategias pertinentes para responder mejor a sus necesidades institucionales y educativas. Es importante señalar que este programa atendió el año 2005, a un total de 443 liceos, localizados en todas las regiones del país.

El año 2005 se realizó un nuevo estudio sobre la situación de los establecimientos que impartían enseñanza media y se logra identificar a 727 establecimientos educativos que se diferencian radicalmente del resto de los centros educativos subvencionados del país, y que requieren, por tanto, de una estrategia especial que les permita mejorar sus resultados de aprendizaje.

En el año 2010, aparece como un antecedente relevante, que, en el marco de la política de descentralización chilena, se aprueba la creación de una Agencia de Calidad, abordándose no sólo la medición de aprendizajes, sino también el diseño de estándares para evaluar el desempeño de sostenedores, docentes y directivos. Al respecto, centros avanzados en educación, como es el Centro de Estudios de Políticas y Prácticas Educativas (CEPPE) de Chile, tiene entre sus líneas prioritarias la investigación sobre liderazgo escolar, poniendo un acento especial en saber quiénes son los actores claves de los centros educativos, cuáles son sus vocaciones, cómo se distribuyen por género, cuáles son sus atribuciones, según dependencia, cuáles son sus trayectorias de vida, y lo más relevante, cómo impacta el trabajo de los directores en las prácticas docentes.

Por su parte, el Centro de Innovación en Educación de Fundación Chile (CIE) se ha orientado en aportar a una mejor comprensión del sistema escolar chileno, sistematizando evidencia que contribuye a la toma de decisiones en materia de políticas educativas en Chile. En este contexto, el Estudio Comparado de Liderazgo Escolar: Aprendizajes para Chile a partir de los Resultados PISA 2009" (Marfán, 2012) da cuenta de la configuración de un conjunto de factores que contribuye a delinear el liderazgo escolar en Chile, en comparación con los demás países que rinden pruebas del Programa Internacional para la Evaluación de Estudiantes (PISA) en el año 2009. El estudio profundiza en el ejercicio del liderazgo en Chile, indagando en los factores que afectan su ejecución y en cómo inciden en los resultados de aprendizaje de los estudiantes. Para ello, se considera un modelo de relación indirecta entre prácticas de liderazgo y logros académicos. Los resultados indican que el liderazgo tiene un efecto sobre los resultados en lectura, aunque bajo, siendo su principal vía 
de influencia el efecto sobre factores de los docentes que inciden en el clima de aprendizaje en el aula.

Otro estudio, realizado en el año 2011, denominado "Formación y Entrenamiento de los Directores Escolares en Chile: Situación actual, desafíos y propuestas de política" (Muñoz y Marfán, 2011), caracteriza los directores en Chile, identificando el liderazgo efectivo como una competencia fundamental. La investigación también ofrece propuestas de política orientadas a mejorar la formación de directores.

Considerando lo antes expuesto, la Fundación Chile, con la participación y colaboración de directivos y especialistas de todo el país, y tomando como referente a su vez, el Marco para la Buena Dirección, establece tres ámbitos de gestión orientadores de la actividad directiva: gestión institucional, gestión curricular y la gestión de la convivencia escolar.

En este contexto, cada uno de los antecedentes expuestos, contribuye a la comprensión de la problemática en estudio, principalmente porque una de las prioridades del MINEDUC se centra en mejorar la calidad de la enseñanza y de los aprendizajes. Es ahí donde las buenas prácticas de gestión al interior de las comunidades educativas cobran sentido.

Por todo esto, el presente trabajo plantea como objetivo general, conocer las percepciones de los agentes integrantes acerca de las políticas de mejoramiento de la calidad de la gestión escolar. Y como objetivos específicos pretende, en primer lugar, describir cómo se vivencian las estrategias de mejoramiento ministeriales al interior de la comunidad educativa; en segundo lugar, identificar las percepciones de los docentes y de los estudiantes, en relación con el discurso oficial y con la práctica en los ámbitos relativos a la gestión curricular y gestión para la convivencia e inclusión; y en tercer lugar, conocer las percepciones de docentes, estudiantes, apoderados y directivos en torno a la práctica, respecto al PEI y propuesta educativa, gestión directiva del establecimiento, labor técnico-pedagógico del profesor, capacidades y proyecciones de los estudiantes, convivencia e inclusión vivenciada en la unidad estudiada.

\section{Marco metodológico}

\subsection{Diseño del estudio}

El presente estudio analiza, desde un enfoque complementario y descriptivo, la perspectiva de los docentes, apoderados, estudiantes y directivos, en lo concerniente a los ámbitos del currículum; gestión directiva, y gestión para la convivencia e inclusión versus lo que se evidencia en la práctica del establecimiento educacional en estudio.

\subsubsection{Participantes e instrumentos}

En el momento del estudio, la estructura organizacional del Liceo, estaba compuesta por un Director, un (1) Inspector General, un (1) jefe UTP, (1) jefe de Producción, dos (2) Orientadoras, cuarenta y tres (43) docentes contratados con un total de 1334 horas. La dotación de Asistentes de la Educación, la componen un (1) asis- 
tente de estudiantes, seis (6) administrativos, seis (6) auxiliares y seis (6) para-docentes, cada uno de ellos contratados con cuarenta y cuatro (44) horas.

La muestra para el estudio cuantitativo se conformó por 287 estudiantes, correspondientes a la totalidad de estudiantes de los cursos de $1^{\circ}, 2^{\circ}$ y $3^{\circ}$ año de Enseñanza Media en carreras de Técnicos de Nivel Medio; y por 26 docentes, de los cuales el $61 \%$ eran hombres y el 39\% mujeres. El 4\% tenía menos de 30 años, el $15 \%$ se ubicaba entre el intervalo 30-40 años, el 19\% entre los 40-50 años y el 62\% más de 50 años. En cuanto a su experiencia docente, el 4\% tenía menos de 5 años, el $15 \%$ en torno a 10 años, el $19 \%$ sobre 20 años y el $62 \%$ más de 20.

Para la parte cualitativa del estudio, se trabajó con 5 estudiantes, 5 apoderados (un representante por cada curso) con 2 años de experiencia, 5 docentes (considerando asistencia al Consejo de Profesores y disponibilidad horaria) con más de 20 años de experiencia y 1 directivo (Director del establecimiento) con 10 años de experiencia en gestión académica.

\subsubsection{Instrumentos}

Para responder a los objetivos de este estudio, se utilizaron dos cuestionarios con tres alternativas de respuestas: Sí, No o No sabe/No responda, con la finalidad de que los encuestados asuman una posición determinada. En este sentido, el cuestionario para estudiantes consta de 40 preguntas cerradas, mientras que el cuestionario para docentes comprende 46 preguntas cerradas. Ambos instrumentos se construyen tomando como referencia la revisión teórica, en lo concerniente a los ámbitos del currículum; gestión directiva, y gestión para la convivencia e inclusión, estipulados por el MINEDUC.

En el caso de los estudiantes, se entrevista a cinco sujetos. También se aplica una entrevista a cinco docentes, cinco apoderados y al director del liceo.

\subsubsection{Validez y fiabilidad}

Los instrumentos son sometidos a un panel de jueces expertos, conformado por tres especialistas, de quienes se recogen y analizan las opiniones especializadas, tomándose las siguientes decisiones: a) se incluyen en el instrumento los ítems en donde hay un $100 \%$ de coincidencia favorable entre los jueces; b) se excluyen los ítems donde hay un $100 \%$ de coincidencia desfavorable; y c) se revisan los ítems en donde hay una coincidencia parcial entre los jueces.

Por su parte, el cálculo de los estadísticos de fiabilidad se realiza a través del método de dos mitades, dividiéndose el test en dos mitades equivalentes hasta hallar la correlación entre éstas. Este método permite elegir los elementos impares del test, para constituir una mitad, y los elementos pares para constituir la otra. Teniendo en cuenta que la literatura define que el valor mínimo aceptable para instrumentos recién desarrollados puede ser de 0,60, los valores de Alfa de Cronbach entre 0,70 y 0,90 indican una buena consistencia interna. Al respecto, los coeficientes Alfa de Cronbach (todas las preguntas) y de dos mitades calculados para los cuestionarios satisfacen dichos valores. 


\section{Resultados}

A modo de síntesis, se presentan los resultados que se obtienen a partir de la triangulación de la información, de los análisis descriptivos y cualitativos de la información.

\subsection{En relación al PEI y propuesta educativa del liceo}

Existe correspondencia entre los resultados de la encuesta y entrevista respecto de la falta de participación de estudiantes, docentes y apoderados en la elaboración e implementación del Proyecto Educativo institucional. Para el caso de los apoderados, manifiestan no conocer en profundidad los aspectos que plantea el PEI. No obstante, se sienten comprometidos con la propuesta educativa que entrega el Liceo, especialmente por la adhesión que sienten hacia el Director.

Respecto de la encuesta aplicada a los docentes, el 92,3\% conoce el PEI del Liceo (Figura 2), aspecto que se relaciona con las entrevistas, no obstante, no se sienten identificados considerando que no han sido parte de la elaboración del mismo, tal como lo expresan un docente entrevistado: "EL PEI lo visualizo como un documento pertinente a la definición del plan de acción del liceo, pero carente de participación de todos los estamentos involucrados en su elaboración, como por ejemplo: estudiantes, apoderados y docentes".

Existe correspondencia entre la encuesta aplicada a 26 docentes y la entrevista donde el $69,2 \%$ de los profesores señala que la Dirección del establecimiento no contribuye a la realización de prácticas pedagógicas de calidad y el $61.5 \%$ señala que la gestión del director no potencia el desarrollo del liceo (Véase la Figura 1). En las entrevistas se hace referencia a la falta de liderazgo, la falta de apoyo a los docentes, entre otros elementos.

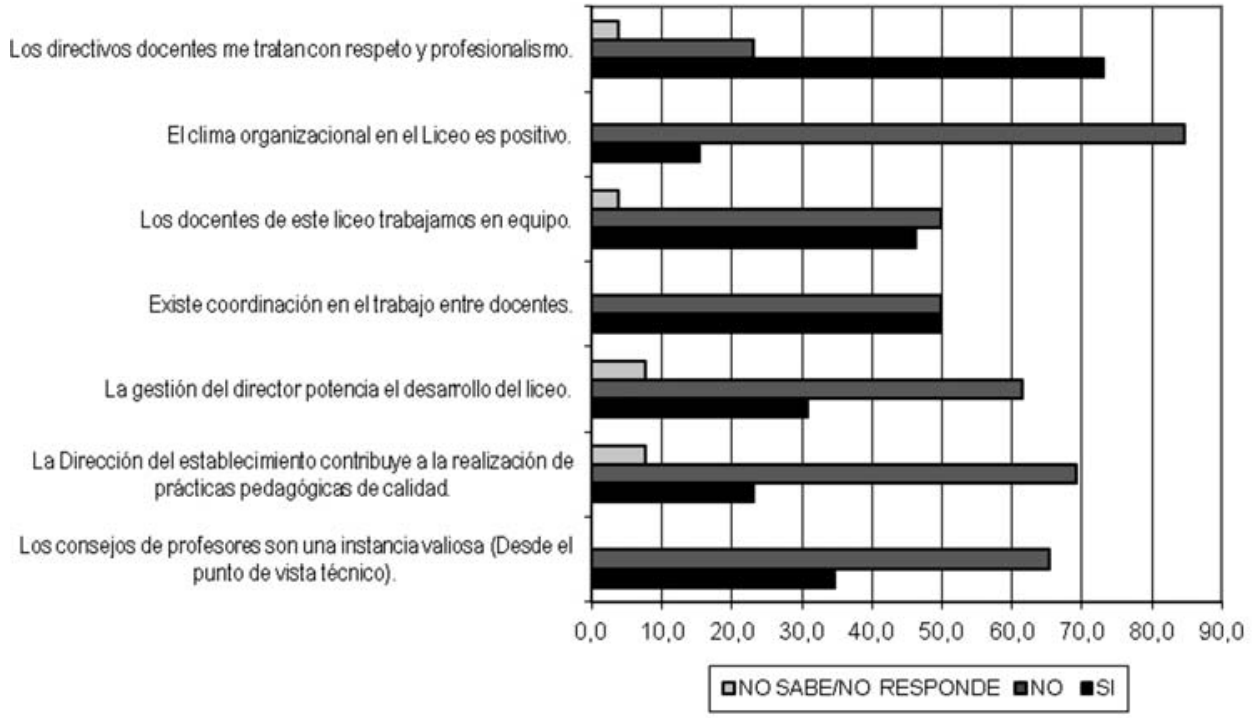

Figura 1. Percepción de los Docentes. Área Gestión Curricular. Gestión Directiva 


\subsection{En relación a la gestión directiva del establecimiento}

Con respecto a esta dimensión, el 88,5\% de los docentes percibe positivamente la modalidad de Grupos Profesionales de Trabajo (GPT), mientras que el 53,8\% vivencia el Proceso de Evaluación Docente con actitud positiva, y el 92,3\% dice conocer el PEl del Liceo (véase la Figura 2).

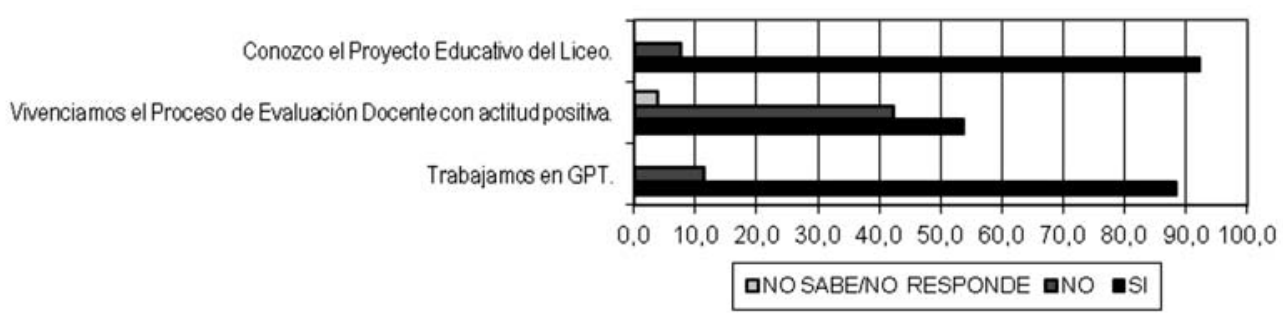

Figura 2. Percepción docente. Gestión directiva

Por otra parte, el Director del liceo destaca en la entrevista aspectos vinculados a la importancia del liderazgo, de ser una persona comprometida con la labor pedagógica; con experiencia docente; con una buena predisposición hacia el trabajo en "terreno" más que de oficina; preocupado por lo humano, lo que se traduce en un compromiso con sus profesores, estudiantes(as) y apoderados con un reconocimiento entre los profesores y superiores directos, de su calidad docente y de manejo administrativo; con una política de "puertas abiertas" para toda la comunidad escolar, profesores, estudiantes y apoderados.

De igual manera, el Marco para la Buena Dirección declara las competencias que deben existir para ejercer una buena dirección. En este contexto, existen cuatro áreas de este marco, como lo son; liderazgo, gestión curricular, gestión de recursos, gestión de clima organizacional y convivencia, con estándares y descriptores que permiten visualizar el deber ser del director.

Finalmente, cabe señalar que el discurso oficial del Ministerio de Educación, respecto de lo que se espera de un director, no se corresponde con las percepciones de la comunidad educativa en estudio, principalmente por los Docentes y alumnos, que manifiestan: "En lo personal, pienso que falta liderazgo y mayor reconocimiento por parte de la comunidad educativa" (Docente); "Falta liderazgo y valoración de los profesores"(Alumno); "La gestión se desarrolla en la medida de las posibilidades del liceo, no olvidemos que este ha tenido una sobrecarga de proyectos que se implementan como Liceo para Todos, Liceos Prioritarios, Formación Dual, etc." (Docente).

Sin embargo, para el caso de los apoderados, estos evidencian una gran valoración hacia el director, con opiniones, tales como: "Confío en la educación que le entregan a los jóvenes y en el Director del Liceo". "El Director es muy buena persona, ayuda mucho a mis dos hijos, ellos almuerzan acá y están cursando una carrera técnica". 


\subsection{Respecto a la gestión técnico-pedagógica del liceo}

Considerando las percepciones de los docentes de la comunidad educativa en estudio, es evidente que este estamento debe cumplir una serie de tareas desde el ámbito técnico pedagógico, por lo que el $92.4 \%$ de ellos reconoce haber presentado su planificación anual. No obstante el 50\% manifiesta que no planifica diariamente, lo que evidentemente podría influir en el desarrollo de prácticas pedagógicas poco pertinentes a la realidad de los estudiantes (véase la Figura 3).

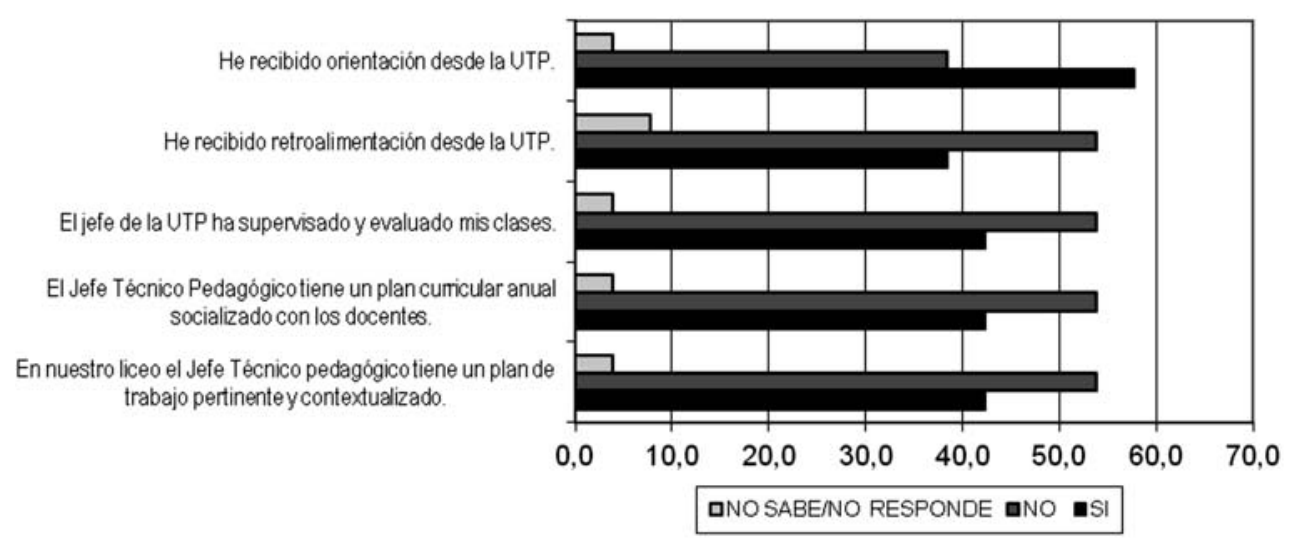

Figura 3. Percepción docente. Área Gestión Curricular. Valoración Gestión Técnico-Pedagógica del Liceo

Por su parte, en las entrevistas, los estudiantes opinan que: "Ha habido talleres de reforzamiento de matemáticas, lo que nos ha servido para mejorar nuestros conocimientos". "Creo que el jefe de UTP tiene que trabajar más en el apoyo de los profesores y estudiantes, ya que el rendimiento de los estudiantes no es bueno."

\subsection{Respecto a la propia labor técnica pedagógica del profesor}

En cuanto a esta dimensión, los docentes perciben que cumplen con la planificación anual al inicio del año; implementan prácticas que permiten crear un ambiente propicio para el aprendizaje; poseen los saberes teórico-práctico, que les permiten comprender la situación socio-educativa de sus estudiantes, y que han participado en la elaboración del PEl. Sin embargo, el 50\% dice no elaborar planificaciones diarias (véase la Figura 4), lo que se contradice con buenas prácticas docentes.

En relación con la revisión de los libros de clase, se evidencia que existe un registro muy acotado de las actividades diarias que se realizan, donde no se logra evidenciar en profundidad el proceso académico de los estudiantes. En este sentido, los docentes entrevistados expresan que "Considero que el trabajo es suficiente, respecto de las condiciones difíciles y todos requerimientos del establecimiento" y que "en la mayoría de los casos la labor pedagógica es adecuada a las exigencias del establecimiento y acotada a las capacidades de los estudiantes", mientras que los apoderados opinan de los profesores que "...son muy buenos, sobre todo los de 
especialidad y otros no tanto como los de matemáticas" y que "Los profesores tienen demandas asociadas a sus remuneraciones y dejan de lado lo principal que tiene relación con mejorar sus capacidades técnicas pedagógicas."

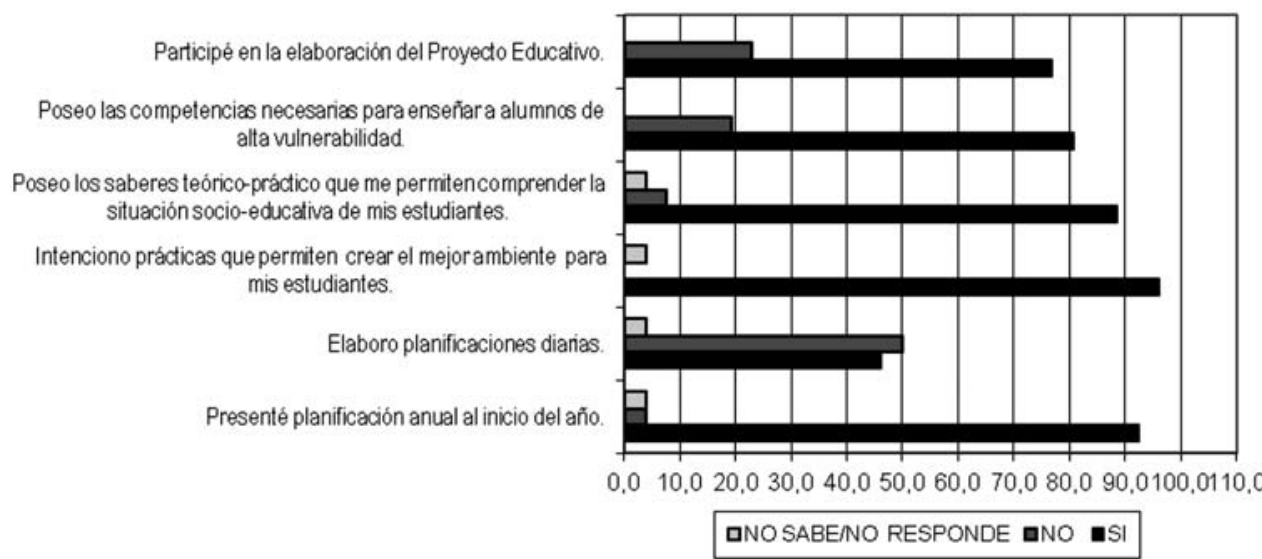

Figura 4. Percepción docente. Área Gestión Curricular. De su propia labor Técnico Pedagógica

\subsection{En relación con las capacidades y proyecciones de los estudiantes}

Las percepciones de los docentes, en relación con las capacidades de sus estudiantes para continuar cursando una carrera técnica en la Educación Superior, son bastante disminuidas, considerando que el 53,8\% considera que los estudiantes estarían en condiciones de lograrlo. De igual manera, solamente el 38,5\% piensa que los estudiantes están capacitados para continuar cursando una carrera profesional en la Educación Superior. Aparece como un elemento relevante que el 76,9\% considera que los estudiantes al finalizar su enseñanza media están capacitados para postular a las escuelas matrices de las Fuerzas Armadas (véase la Figura 5). Este hecho se condice con la idea de que un porcentaje importante de los estudiantes que egresan del liceo postulan a dicha escuela, siendo aceptados.

Cabe señalar que es preocupante que tanto directivos como profesores tengan una valoración disminuida de las características de los estudiantes, mientras que los padres tienen altas expectativas del Liceo, cuando declaran: "Los estudiantes tienen las mismas capacidades y proyecciones que cualquier otro joven, solo depende de ellos". "Los estudiantes salen preparados para el mundo laboral". De la misma manera, un estudiante señala que "Mis proyecciones están orientadas a encontrar trabajo y luego de unos dos años estudiar Mecánica en un Instituto". "Los estudiantes tenemos cada vez más posibilidades de ir a la Universidad o elegir trabajar y luego estudiar una carrera".

Un docente entrevistado evidencia sus pocas expectativas con los estudiantes cuando señala que "Los estudiantes se ven limitados a su desarrollo futuro principalmente por la falta de recursos económicos, sin embargo algunos encuentran bue- 
nas posibilidades de desarrollo personal y profesional en las escuelas matrices de las Fuerzas Armadas y no así para las Universidades o Institutos profesionales".

En la entrevista realizada a los estudiantes, se evidencia carencia en la proyección de estos en el ámbito de la educación superior, no obstante, se visualizan en el campo laboral una vez que hayan finalizado su proceso de formación en el liceo.

Por último, es importante señalar que desde el discurso oficial del MINEDUC, y específicamente desde la Red de Maestros de Maestros, se enfatiza en la importancia de las expectativas de los docentes respecto de los estudiantes. En este contexto, se entiende que el estudiante otorga valor a las opiniones del profesor, por lo que es indispensable que éste valore los logros de los estudiantes y genere expectativas positivas.

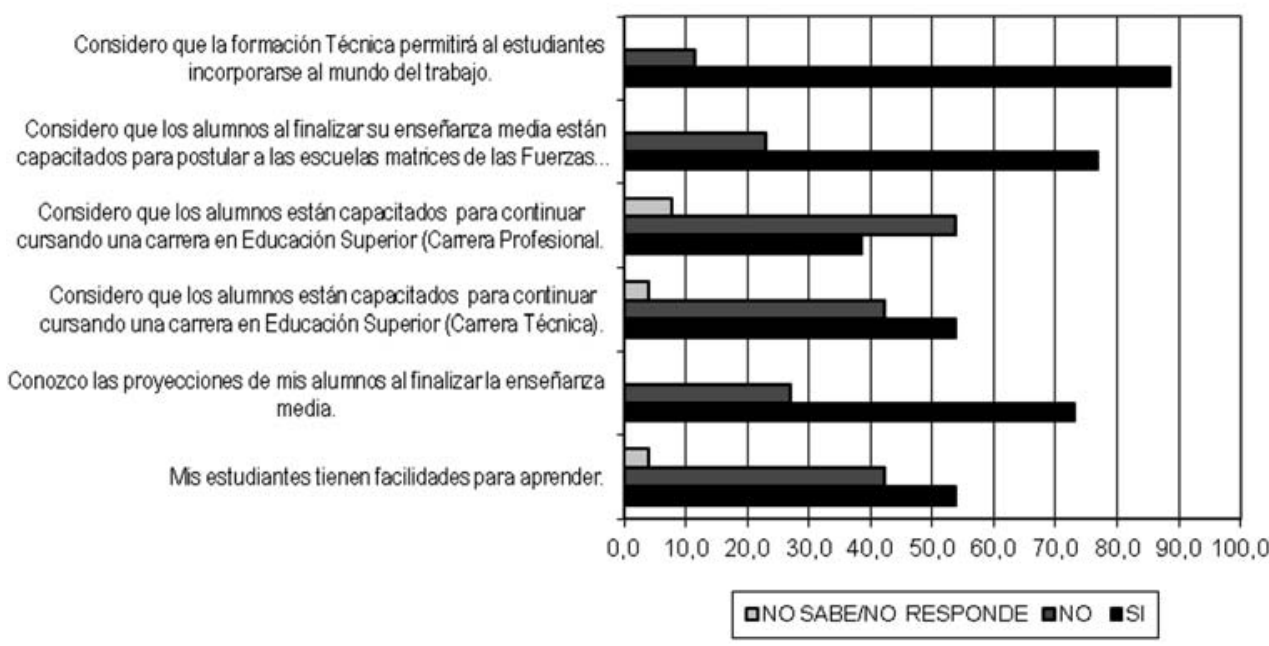

Figura 5. Percepción de los Docentes hacia los estudiantes. Proyecciones

\subsection{En relación con la convivencia e inclusión vivenciada en el liceo}

El $68,6 \%$ de los estudiantes percibe que los profesores no aceptan su música, vestimenta y gustos, y el $50 \%$ que los profesores valoran a los estudiantes y alumnas por igual (véase la Figura 6).

Cabe señalar, que la responsabilidad de la aplicación de la normativa recae en Inspectoría general, una vez que los casos más complejos superan las estrategias aplicadas por el profesor. Estos declaran que los problemas disciplinarios muchas veces son tratados por orientación o por el mismo director, quién manifiesta: "Considero que esto se maneja de buena forma a través de la Inspectoría General como primera instancia y esta Dirección como instancia resolutiva. Por otro lado, existe un Manual de convivencia que regula estos aspectos".

Por su parte, los estudiantes y apoderados perciben que: "La convivencia en general es buena, pero con dificultades de vez en cuando". 


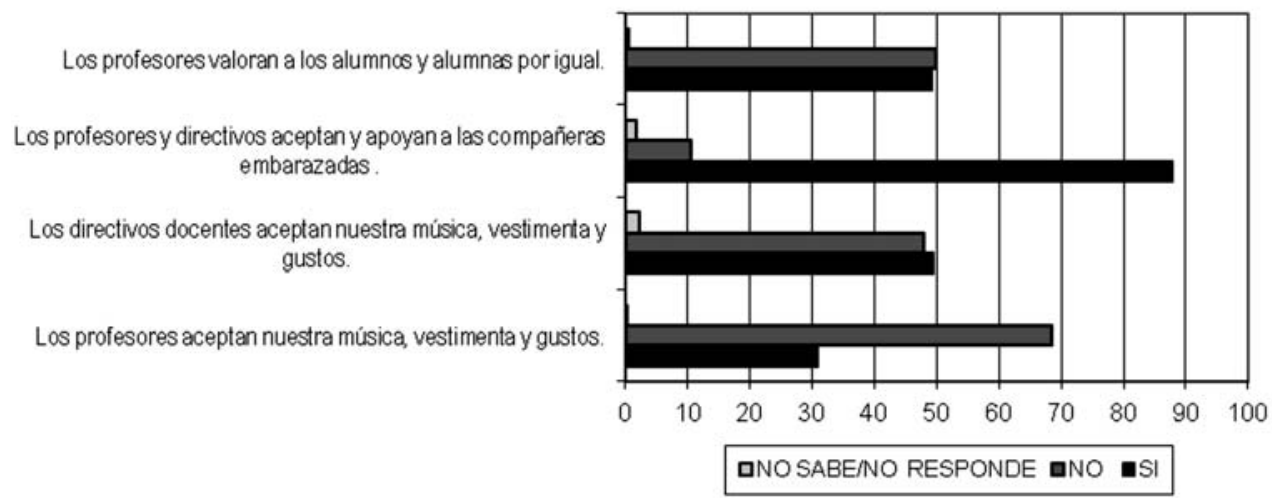

Figura 6. Percepción de los Estudiantes. Área Convivencia Escolar. Gestión Directiva

\subsection{En relación con los espacios de participación}

Con respecto a esta dimensión, el $80,8 \%$ de los estudiantes señala que no participa en el centro de estudiantes, un $77 \%$ no siente que el Centro de Estudiantes los representa, ni tampoco participan en el consejo escolar (véase la Figura 7). Por su parte, las entrevistas evidencian que las estructuras de participación existen, no obstante, falta desarrollar una plataforma efectiva de trabajo que permita desarrollar un mayor nivel de identidad desde los integrantes de la comunidad educativa. En este contexto, el Director del Liceo manifiesta las dificultades que se presentan a la hora de pretender tomar decisiones incorporando a los diversos integrantes de la comunidad educativa, considerando que no existe una cultura de ello. Por otro lado, el director manifiesta: "el consejo escolar, el centro de estudiantes, de padres y apoderados, son generados por procesos democráticos y la dirección del Liceo deter-

Participo en el consejo escolary otras actividadessimilares

Siento que el Centro de Alumnos me representa

Participo en el Centro de Alumnos

Tengo facilidades para aprender

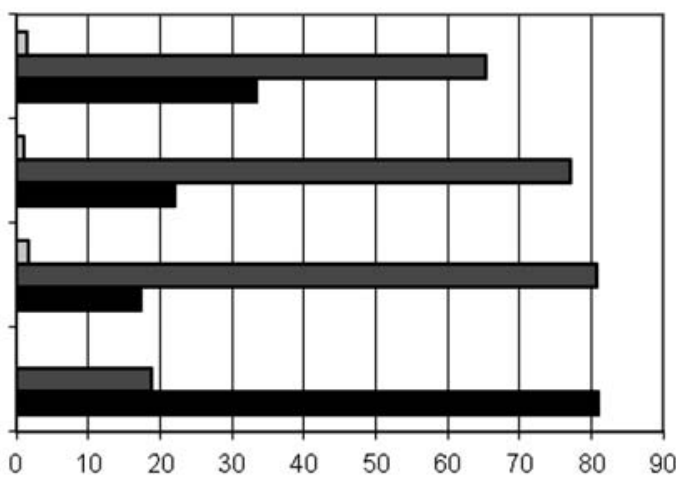

口NOSABE/NO RESPONDE םNO $\mathbf{m S I}$

Figura 7. Percepción de los Estudiantes. Área Convivencia Escolar de su propia participación 
mina espacios y tiempos para el adecuado funcionamiento de dichos estamentos, cuando son necesarios". En este sentido, los docentes plantean que: "la participación es débil, pero se gesta a través del consejo académico, talleres extracurriculares y reuniones de apoderados", a través de las reuniones de profesores y otras actividades que se han desarrollado durante el año".

Por su parte, los apoderados entrevistados valoran las instancias de participación, en este contexto exponen que "El Liceo brinda participación en espacios como reuniones de apoderados y reuniones de centro general de padres y apoderados". Finalmente, los estudiantes reconocen que los espacios de participación son principalmente "... a través de talleres extra programáticos como deporte y computación".

\section{DisCuSión y CONCLUSIONES}

En relación con el objetivo general de investigación orientado a: "Conocer las percepciones de los agentes integrantes acerca de las políticas de mejoramiento de la calidad de la gestión escolar", se concluye que efectivamente, las políticas ministeriales son parte de la definición de políticas de la comunidad educativa, establecidas en el Programa de Desarrollo Institucional, no obstante, en la práctica, se evidencia falta de participación de todos los actores de la comunidad educativa, ya que la estrategia de participación en el Plan Estratégico Institucional, no da cuenta de los intereses de la comunidad.

En relación con el primer objetivo específico orientado a "Describir cómo se vivencian las estrategias de mejoramiento ministeriales al interior de la comunidad educativa", se puede concluir que las percepciones de los agentes integrantes tienen niveles de similitud, al declarar la inefectividad en aspectos pedagógicos que permitan levantar propuestas académicas pertinentes y contextualizadas, con lo cual se puede concluir que no es posible implementar las políticas educativas institucionales, ya que existen otras necesidades básicas insatisfechas y que son consideradas prioritarias para los profesores.

Con respecto al segundo objetivo específico orientado a "Identificar las percepciones de los docentes y de los estudiantes, en relación con el discurso oficial y con la práctica en los ámbitos relativos a la gestión curricular, y gestión para la convivencia e inclusión", se concluye que los docentes están cansados de los proyectos que instala el MINEDUC en las comunidades educativas, como por ejemplo, Proyecto Liceo para Todos y Liceos Prioritarios.

En el contexto de la toma de decisiones curriculares, se puede concluir que, aunque las estructuras de participación existen, falta proporcionar una plataforma efectiva de trabajo que permita desarrollar un mayor nivel de identidad en los miembros de la comunidad educativa. Por ello, es evidente la dificultad que se presenta a la hora pretender tomar decisiones, incorporando a los diversos miembros de la comunidad educativa.

En el ámbito de la gestión de la convivencia se concluye que si bien existe un Manual de Convivencia, no existe una estructura que permita sustentar su aplicación. Además, se concluye que no todos los estudiantes conocen este manual, generando una práctica correctiva generalmente establecida a criterio del Inspector 
General o el Director. De este análisis se infiere que los conflictos al interior de la comunidad educativa no se resuelven adecuadamente o no existen los mecanismos adecuados para abordarlos, debilitando así el cumplimiento con las metas del PEI y afectándose además la comunicación interna.

Finalmente, en relación con el tercer objetivo específico relacionado con "Conocer las percepciones de docentes, estudiantes, apoderados y directivos en torno a la práctica, respecto al PEI y propuesta educativa, gestión directiva del establecimiento, labor técnico-pedagógico del profesor, capacidades y proyecciones de los estudiantes, convivencia e inclusión vivenciada en la unidad estudiada", se concluye que casi la totalidad de los docentes considera que la formación técnica entregada por el Liceo permite al estudiante incorporarse al mundo del trabajo. También se concluye que debe darse énfasis a los mecanismos de apoyo y seguimiento a los estudiantes, especialmente a los más desfavorecidos, de manera de generar las condiciones adecuadas para el cumplimiento del PEl y las políticas públicas. Por otro lado, se concluye que es importante seguir potenciando el trabajo de orientación focalizado fundamentalmente en los problemas sociales, que afectan a los estudiantes, a través de la línea de orientación vocacional.

Cabe señalar, que la micro implementación del currículum no necesariamente se ajusta al discurso ministerial. Por ello, se hace necesario flexibilizar el currículum de formación en función de las variables internas y externas, lo cual permitiría a la Unidad Técnico Pedagógica, una planificación con mayor pertinencia curricular. El equipo directivo no tiene un plan de trabajo anual orientado a evaluar la implementación del currículum y a desarrollar las acciones de mejora necesarias para lograr los objetivos planteados, mas bien se rige solamente por el calendario académico, lo que refleja una falta de rigurosidad evidente en la gestión académica, que conlleva a una falta de liderazgo por parte de la encargado de esta unidad, quien debe llevar adelante las estrategias asociadas al currículum de formación.

Una de las grandes debilidades detectadas durante la investigación recae en la Unidad Técnico Pedagógica, donde se evidencia que no existe una planificación pertinente y contextualizada. Al respecto, la mayoría de los docentes señala que no existe un plan curricular socializado, y otros tantos señalan que hace falta supervisión y evaluación de las clases. En este sentido, se puede concluir que esta carencia se genera a partir de la carga administrativa demandada por el MINEDUC y la Corporación Municipal de Educación hacia esta unidad, lo que le impide disponer de más y mejores espacios de apoyo técnico-profesional.

Existe carencia de recursos pedagógicos en el área técnica, lo que debilita el cumplimiento del PEI, en su conjunto, generando, de este modo, dificultades en su implementación y en su seguimiento, lo cual atenta directamente contra la política pública ministerial.

Adicionalmente, y dado que un tercio del alumnado manifiesta no conocer o no asistir a los talleres extracurriculares, y un cuarto señala no conocer o no participar en programas de nivelación para estudiantes con bajo rendimiento, se concluye que existe un importante número de estudiantes que no participa o no conoce las actividades complementarias que ofrece la institución, lo cual refleja desconocimiento de las actividades o falta de motivación. Esto atentaría directamente contra el logro 
de los objetivos del proyecto educativo y principalmente los aprendizajes de los estudiantes. Por último, es importante señalar que desde el discurso oficial del MINEDUC, y específicamente desde la Red de Maestros de Maestros, se enfatiza en la importancia de las expectativas de los docentes en relación con el desempeño académico de los estudiantes. En este contexto, se entiende que el estudiante otorgue valor a las opiniones y expectativas del profesor, por lo que es indispensable que éste valore los logros de los estudiantes y genere altas expectativas académicas en ellos.

\section{IMPLICACIONES}

Desde el punto de vista teórico, este estudio ofrece como principal contribución, la propuesta de un modelo para evaluar la gestión de un establecimiento educacional en sus distintos ámbitos, según las categorías definidas por el propio MINEDUC. Por otra parte, una segunda contribución teórica, radica en la identificación de un conjunto de variables contextuales, cuyo desarrollo puede contribuir a que la institución estudiada establezca una cultura de la participación, que ayude a sustentar las diversas acciones del Proyecto Educativo Institucional. Otra importante implicación teórica, es que este trabajo genera la necesidad de elaborar una estrategia de liderazgo distribuido que permita incorporar a los diversos agentes integrantes en la toma de decisiones.

Por otra parte, la repercusión práctica más evidente del presente estudio, radica en la posible utilización de los resultados para la toma de decisiones, en materia de gestión directiva, en la institución educativa estudiada. En este sentido, podría servir como un diagnóstico organizacional orientado a mejorar el desempeño institucional en el ámbito de la gestión directiva y curricular. Además, los resultados obtenidos podrían servir como insumos para la reflexión acerca de la importancia que tienen las variables contextuales, tales como la cultura organizacional, el trabajo en equipo, la autonomía y el aprendizaje organizacional para el logro de los objetivos planteados en el Proyecto Educativo Institucional.

Finalmente, y tras realizar las adaptaciones que sean necesarias, la metodología diseñada para este estudio podría ser utilizada en procesos de autoevaluación en otras unidades educativas ampliándose, de esta manera, la utilidad de la misma.

\section{REFERENCIAS BIBLIOGRÁFICAS}

BARRIO, B. (2006). Calidad, Equidad e Indicadores en el Sistema Educativo Español. Pulso: revista de educación, 29, 43-58. Disponible en: http://dialnet.unirioja.es/ descarga/articulo/2200891.pdf.

DARLING-HAMMOND, L. (1999). Teacher Quality and Student Achievement: A Review of State Policy Evidence. Center for the Study of Teaching and Policy. University of Washington. Disponible en: http://depts.washington.edu/ctpmail/ PDFs/LDH_1999.pdf.

DARLING-HAMMOND, L. y ROTHMAN, R. (2011). Teacher and Leader Effectiveness. High-Performing Educations Systems. Stanford Center for Opportunity Policy and Education. Disponible en: http://www.all4ed.org/files/TeacherLeader EffectivenessReport.pdf. 
ANÁLISIS DE LA GESTIÓN DE LA POLÍTICA EDUCATIVA DEL MINISTERIO DE EDUCACIÓN DE CHILE...

FULLAN, M. (1993). Why Teachers must become Change Agents? Educational Leadership, 50 (6). Disponible en: http://www.michaelfullan.ca/media/13396031680.pdf.

FULLAN, M. y HARGREAVES, A. (2012). Reviving Teaching with Professional Capital. Education Week. Disponible en: http://www.edweek.org/ew/articles/2012/ 06/06/33hargreaves_ep.h31.html.

MARFÁN, J. (2012). Estudio comparado de liderazgo escolar: Aprendizajes para Chile a partir de los resultados PISA 2009. Fondo de Investigación y Desarrollo en Educación - FONIDE. Departamento de Estudios y Desarrollo. División de Planificación y Presupuesto.

MINEDUC (2005). Marco para la Buena Dirección. Criterios para el Desarrollo Profesional y Evaluación del Desempeño. Publicación de la Unidad de Gestión y Mejoramiento Educativo. División General de Educación. Disponible en: http:// ww2.educarchile.cl/UserFiles/P0037/File/Directores/15.si\%20Marco_para_la_ Buena_Direccion.pdf.

MINEDUC (2008). Características de un Buen Proceso de Elaboración del Plan de Mejoramiento Educativo. Disponible en: http://www.planesdemejoramiento.cl/ m_anexos/Anexo\%20V.pdf.

MORIN, E. (1999). Seven complex lessons in education for the future. UNESCO. Disponible en: http://unesdoc.unesco.org/images/0011/001177/117740eo.pdf.

MUÑOZ, G. y MARFÁN, J. (2011). Formación y entrenamiento de los Directores Escolares en Chile: Situación actual, desafíos y propuestas de política. Fondo de Investigación y Desarrollo en Educación - FONIDE. Departamento de Estudios y Desarrollo. División de Planificación y Presupuesto. Disponible en: http:// educacionfch.cl/nuestro-centro/que-hacemos/estudios-cie/47-centro-deinnovacion/estudios/archivo-estudios/307-formacion-y-entrenamiento-de-losdirectores-escolares-en-chile-situacion-actual-desafios-y-propuestasde-politica.html.

TOBÓN, S.; SÁNCHEZ, A.; CARRETERO, M. A. y GARCÍA, J. A. (2004). Calidad y Educación Superior. (1aㅡ ed.). Bogota: Cooperativa Editorial Magisterio, 2004.

UNESCO (2000). Marco de Acción de Dakar. Disponible en: http://unesdoc.unesco. org/images/0012/001211/121147s.pdf.

UNESCO (2008). Informe de Seguimiento de la Educación para Todos (EPT) en el Mundo. Disponible en: http://unesdoc.unesco.org/images/0017/001776/177609s.pdf.

VALDEBENITO, L. (2011). La Calidad de la Educación Chile: ¿Un Problema de Concepto y Praxis? Revisión del Concepto de Calidad a partir de Dos Instancias de Movilización Estudiantil (2006 y 2011). Revista del Centro Telúrico de Investigaciones Teóricas. Disponible en: http://www.cisma.ctit.cl/1\%20numero/ Ivaldebenito-calidad.pdf. 